the past three decades in India. The initial experiments' started in 1981 with a circus tragedy and documentation of prolonged grief reaction. In the Bhopal gas tragedy (1984) mental health services were integrated through primary care doctors. The Marathwada earthquake (1991) involved primary health care personnel in provision of mental health care to the survivors. The Orissa super cyclone (1999) saw the emergence of psychosocial support to the community using local resources like community level workers who were survivors by themselves. The feasibility study involving 40 such workers was expanded to a pilot model with 400 workers in the Gujarat earthquake (2001) and later to the level of a District model in the Gujarat riots (2002). These developments paved way for the State model when Tsunami struck the eastern coast of India affecting three States and two Union Territories in India. The experiences and experiments led to the development of standardized capacity building tools and intervention kits with level and limits of care being addressed. The Indian experiences has seen a striding change from psychiatry paradigm to public health model, to the development of a standardized psychosocial support models involving community at large. The lesson learnt has been helpful in developing the National Guidelines on Psychosocial Support and Mental Health Services by the National Disaster Management Authority of India. These service models could be adapted to the developing South East Asian countries where there is a paucity of trained professionals to attend the needs of the survivors.

Prehosp Disaster Med 2011;26(Suppl. 1):s3-s4

doi:10.1017/S1049023X11000276

(A15) Trauma Signature Analysis: Evidence-Based Guidance for Disaster Mental Health Response

J.M. Shultz, ${ }^{1}$ Y. Neria, ${ }^{2}$ Z. Espinel, ${ }^{3}$ F. Kelly ${ }^{4}$

1. Center for Disaster \& Extreme Event Preparedness, Miami, United States of America

2. New York State Psychiatric Institute, New York City, United States of America

3. Center for Disaster and Extreme Event Preparedness, Miami, United States of America

4. Dublin, Ireland

Introduction: The first decade of the 2000s has advanced the field of mental health and psychosocial support (MHPSS) in disasters by providing expert consensus guidance. Nevertheless, MHPSS response to major disasters is frequently uncoordinated and rarely based on scientific evidence. Moreover, MHPSS response is not customized to the unique constellation of stressors and psychological risk factors that distinguish each disaster event. To address this lack of science and specificity, we have developed trauma signature (TSIG) analysis.

Methods: TSIG analysis consists of the following steps. Risk factors for disaster-related psychological distress and psychopathology (e.g., PTSD, depression) are continuously documented, updated, and refined. When disaster strikes, situation reports (sitreps) are issued in the early aftermath. We examine initial sitreps to determine the presence and intensity of evidencebased risk factors, subsumed under the headings of exposure to hazards, loss, and change. We estimate the size of the affected population. We rapidly create an initial TSIG and translate findings into actionable guidance regarding probable MHPSS needs for services and personnel.
Results: We have constructed TSIGs for prominent 2010 disasters: Haiti earthquake, Deepwater Horizon oil spill, and Pakistan monsoonal flooding. Psychological risk factor profiles contrast sharply across these three salient events. Regarding exposure to hazards, numbers of persons experiencing physical injury and perceiving threat to life are highly divergent. Losses differ dramatically when quantified in terms of deaths, numbers bereaved, homes and livelihoods lost, and economic toll. The degree of lifestyle and societal change, including displacement, lack of survival needs, lack of security, and interpersonal violence, also differentiates the psychological impact of these disparate events.

Conclusion: TSIG analysis can be used to provide rapid postimpact/pre-deployment MHPSS response guidance based on risk factor assessment. Using TSIG analysis, MHPSS response can be tailored and timed to the defining features of the disaster event.

Prehosp Disaster Med 2011;26(Suppl. 1):s4

doi:10.1017/S1049023X11000288

\section{(A16) Using Geographical Information Systems in Road Traffic Injury Research: A Case Study of New Mumbai, India \\ A. Srikanth, ${ }^{1}$ B. Guru ${ }^{2}$ \\ 1. Jamsetji Tata Center for Disaster Management, Mumbai, India \\ 2. Tata Institute of Social Sciences, Mumbai, India}

Introduction: A multidisciplinary approach with Geographical information systems, Public health and Social science inputs was adopted to survey the fatal and non-fatal traffic crash events in the New Mumbai municipality region in Western India to identify the risky zones on the arterial highways.

Methods: A Standardized questionnaire was used to collect event data about the time, date, day of the week, location, type of injury, and vehicle type involved in the accidents, from the police station records. The data for the time period of January 2009 to July 2010 was merged into ESRI's ArcGIS software as attribute data. All the crash sites were georeferenced into the base map (with the major road networks of the region) by using a GPS receiver.

Results: Analysis was done for Hot spot identification along the major highways, number of accidents, number of fatalities and injured, case-fatality ratio and number of accidents with only financial loss. Further, the role of environmental, geographical, sociological and constructional factors was highlighted on the locations of the RTC. These roadway factors, weather, population density, road conditions, profile of the injured and healthcare access was studied. Majority of RTCs occurred during normal weather and road conditions, during daylight and on dry roads. All the analyses and interpretations were done within the ArcGIS software environment and classifying RTCs according to the attributes on the Geodatabase gave significant results.

Conclusion: Spatial analysis using GIS for Road Traffic Accidents to identify hot spots to identify high risk zones in the region enables policy makers to design injury prevention strategies for RTCs. In India, further GIS-based research is needed for planning access to emergency health care, to determine environmental-related causes, developing Injury 
registries and design population-based educational interventions in a developing country setting.

Prehosp Disaster Med 2011;26(Suppl. 1):s4-s5

doi:10.1017/S1049023X1100029X

(A17) Epidemiology of Non-Vehicular Trauma Patients in the Prehospital Setting in India

N.A. Lodhia, ${ }^{1}$ M. Strehlow, ${ }^{1}$ E. Pirrotta, ${ }^{1}$

B.N.V. Swathi, ${ }^{2}$ A. Gimkala, ${ }^{2}$ S. Sistla, ${ }^{2}$

R. Rao, ${ }^{2}$ S. Mabadevan ${ }^{1}$

1. Emergency Medicine, Stanford, United States of America

2. Online Medical Research, Secunderabad, India

Background: Non-vehicular trauma (NVT) accounts for $8 \%$ of all calls to the GVK Emergency Management and Research Institute (EMRI), which provides prehospital emergency care to 85 million residents of Andhra Pradesh, India. This study describes the characteristics and outcomes of patients with NVT transported by GVK EMRI.

Methods: All patients with NVT were prospectively enrolled over 28 12-hour periods (equally distributed over each hour of the day and day of the week) during July/August 2010. Patients not found at the scene, refusing service, or reporting self-inflicted injuries were excluded. Real-time demographic and clinical data were collected from prehospital care providers using a standardized questionnaire. Follow-up patient information was collected at 48-hours and 30-days following injury.

Results: A total of 1,569 patients were enrolled. Follow-up rates were $72 \%$ at 48 hours and $71 \%$ at 30 days. The mean patient age was $40(\mathrm{SD}=18)$ and $67 \%$ were male. Adults (ages 18-64) accounted for most patients (80\%), followed by elderly (age $>$ $64,12 \%$ ) and children (age $<18,8 \%$ ). Of the patients, $71 \%$ were from rural/tribal areas and $89 \%$ from lower socioeconomic strata. Eighty-two percent called within 1 hour of injury. Median call-to-scene time was 19 minutes $(\mathrm{SD}=15)$ and sceneto-hospital time was 25 minutes $(\mathrm{SD}=21)$. Most patients suffered blunt injuries (85\%) with falls accounting for $43 \%$ of all injuries. Of the injuries, $56 \%$ were accidents and $43 \%$ assaults. Most injuries involved head/neck (48\%) and extremities (44\%). Cumulative mortality rates prior to hospital arrival, at 48-hours and at 30 -days were $1.1 \%, 3.2 \%$, and $4.9 \%$ respectively. Falls accounted for $69 \%$ of all deaths. Falls and age $>65$ were predictors of mortality $(p<0.0001)$. Of NVT survivors, $56 \%$ returned to baseline function and $28 \%$ were in significant pain or bed bound at 30-days post-injury.

Conclusion: This initial study of prehospital NVT patients in India reveals that falls and elderly age were highly associated with death.

Prehosp Disaster Med 2011;26(Suppl. 1):s5

doi:10.1017/S1049023X11000306

(A18) The Influence of Status and the Patterns of Driving License Ownership Toward the Gradation of Open Fractures According to Sardjito Scoring System Suffered by Motorcycles Accident Patients in Emergency Department of Saiful Anwar General Hospital from April to June 2010 T. Maharani, ${ }^{1}$ A. Haedar ${ }^{2}$

1. Kediri, Indonesia

2. Department of Emergency Medicine, Malang, Indonesia
Introduction: 3880 fracture patients are admitted in the Emergency Department of Saiful Anwar General Hospital from January to August 2009. It signifies to the second place of the cause of patients' admission. Most cases are motorcyclist victims from road traffic accident. Driving license is encouraged by the government to reduce the number of road traffic accident victims.

Methods: This study utilizes observational with cross sectional study and purposive sampling to correlate the relationship between status of motorcycle driving license ownership and the obedience of traffic law, the relationship between the patterns of motorcycle driving license ownership and the obedience of traffic law, and the relationship between the obedience towards traffic law and the gradation of open fractures among motorcyclist victims in accordance with Sardjito Scoring system.

Results: Most patients have no driving license. And those who has driving license, mostly have never undergone driving license test. Patients with open fractures of cruris come with severe Sardjito scoring system, open fractures of antebrachii with moderate Sardjito scoring system, and open fractures of femur with moderate Sardjito scoring system.

Discussion: It is strongly related between the ownership of driving license and road traffic accidents. The most road traffic accidents cases of are open fractures of cruris, open fractures of antebrachii, and open fractures of femur respectively. High obedience and strict use of personal protective equipment (safety helmet, glove, and jacket) would be effective in mitigating road traffic accident injuries.

Prehosp Disaster Med 2011;26(Suppl. 1):s5 doi:10.1017/S1049023X11000318

(A19) Establishing a Trauma Registry at the National Referral Hospital in Thimpu, Bhutan

T.B. Nelp, ${ }^{1}$ N.F. Manice, ${ }^{2}$ S.C. Morris ${ }^{3}$

1. Washington DC, United States of America

2. Washington, DC, United States of America

3. Brigham and Women's Hospital, Boston, United States of America

Background: The burden of traumatic injuries is increasing in Bhutan. Data from the Ministry of Health of Bhutan $(\mathrm{MoH})$ indicates that the number of injuries has increased $37 \%$ from the years 2004 to 2008. Current data on demographics, cause, and outcome of injuries is not well documented, leaving the $\mathrm{MoH}$ with insufficient data to guide policy decisions. The $\mathrm{MoH}$ and the Bhutan Foundation have prioritized development of a national trauma registry in Bhutan, starting with a trauma registry at Jigme Dorji Wangchuck National Referral Hospital (JDWNRH).

Objective: To design and implement a sustainable tool for the collection and storage of data describing trauma patients at JDWNRH.

Design and Methods: The trauma team defined trauma as any injury that requires an evaluation, intervention, or admission to the hospital. A paper based tool was designed by consensus to collect data on demographics, injury type, injury location, injury severity, treatments and outcomes. A hospital based system to processes the data into Microsoft Access was established and data collection began in September 2010. Monitoring is ongoing to ensure the reliability of data. 